\title{
Prospective intraindividual comparison between respiratory-triggered balanced steady-state free precession and breath-hold gradient-echo and time-of-flight magnetic resonance imaging for assessment of portal and hepatic veins
}

\author{
Willmann, J K ; Göpfert, K ; Lutz, A M ; Nanz, D ; McCormack, L ; Petrowsky, H ; Seifert, Burkhardt
} ; Hervo, P ; Marincek, B ; Weishaupt, D

\begin{abstract}
The purpose of this study was to compare respiratory-triggered balanced steady-state free precession (bSSFP) with breath-hold contrast-enhanced dynamic two-dimensional (2D) gradient-echo (GRE) and time-of-flight (TOF) magnetic resonance imaging (MRI) for portal and hepatic vein visualization and assessment of portal and hepatic venous variants. Sixty patients with liver disease underwent nonenhanced bSSFP and contrast-enhanced GRE, bSSFP, and TOF imaging. Contrast-to-noise ratios (CNRs) for portal and hepatic veins were measured. Two readers rated the quality of portal and hepatic vein visualization on a 5-point Likert scale. The diagnostic performance of each MRI series in the detection of portal and hepatic venous variants was assessed in 40/60 patients who also underwent contrast-enhanced multidetector-row computed tomography (MDCT). CNRs for portal and hepatic veins were highest on contrast-enhanced bSSFP images. Image quality of portal and hepatic veins was rated higher for nonenhanced bSSFP than for contrast-enhanced GRE $(\mathrm{p}<0.03)$ and TOF $(\mathrm{p}<0.003)$ and higher for contrast-enhanced than for nonenhanced bSSFP $(\mathrm{p}<0.003)$. Compared with MDCT, portal and hepatic venous variants were identified with an accuracy of $99 \%$ on bSSFP images, with an excellent interobserver agreement (kappa=0.97). Compared with MDCT, presence of surgically important portal and hepatic venous anatomical variants can be predicted with high accuracy on bSSFP images.
\end{abstract}

DOI: https://doi.org/10.1007/s00330-006-0305-3

Posted at the Zurich Open Repository and Archive, University of Zurich ZORA URL: https://doi.org/10.5167/uzh-18203

Journal Article

Published Version

Originally published at:

Willmann, J K; Göpfert, K; Lutz, A M; Nanz, D; McCormack, L; Petrowsky, H; Seifert, Burkhardt; Hervo, P; Marincek, B; Weishaupt, D (2007). Prospective intraindividual comparison between respiratorytriggered balanced steady-state free precession and breath-hold gradient-echo and time-of-flight magnetic resonance imaging for assessment of portal and hepatic veins. European Radiology, 17(1):229-240.

DOI: https://doi.org/10.1007/s00330-006-0305-3 
Jürgen K. Willmann

Kerstin Göpfert

Amelie M. Lutz

Daniel Nanz

Lucas McCormack

Henrik Petrowsky

Burkhardt Seifert

Patrice Hervo

Borut Marincek

Dominik Weishaupt

Received: 20 February 2006

Revised: 4 April 2006

Accepted: 18 April 2006

Published online: 16 May 2006

(C) Springer-Verlag 2006

J. K. Willmann $(\bowtie) \cdot$ K. Göpfert ·

A. M. Lutz · B. Marincek .

D. Weishaupt

Institute of Diagnostic Radiology,

University Hospital Zurich,

Rämistrasse 100 ,

8091 Zurich, Switzerland

e-mail: willmann@stanford.edu

Tel.: +41-44-2553410

Fax: +41-44-2554443

D. Nanz

Department of Medical Radiology, University Hospital Zurich,

Zurich, Switzerland

L. McCormack · H. Petrowsky

Department of Visceral and

Transplantation Surgery,

University Hospital Zurich,

Zurich, Switzerland

B. Seifert

Department of Biostatistics,

University of Zurich,

Zurich, Switzerland

P. Hervo

GE Healthcare,

Buc Cedex, France

\section{Prospective intraindividual comparison between respiratory-triggered balanced steady-state free precession and breath-hold gradient-echo and time-of-flight magnetic resonance imaging for assessment of portal and hepatic veins}

$(p<0.03)$ and TOF $(p<0.003)$ and higher for contrast-enhanced than for nonenhanced bSSFP $(p<0.003)$. Compared with MDCT, portal and hepatic venous variants were identified with an accuracy of $99 \%$ on bSSFP images, with an excellent interobserver agreement $(\kappa=0.97)$. Compared with MDCT, presence of surgically important portal and hepatic venous anatomical variants can be predicted with high accuracy on bSSFP images. TOF imaging. Contrast-to-noise ratios (CNRs) for portal and hepatic veins were measured. Two readers rated the quality of portal and hepatic vein visualization on a 5-point Likert scale. The diagnostic performance of each MRI series in the detection of portal and hepatic venous variants was assessed in $40 / 60$ patients who also underwent contrast-enhanced multidetector-row computed tomography (MDCT). CNRs for portal and hepatic veins were highest on contrastenhanced bSSFP images. Image quality of portal and hepatic veins was rated higher for nonenhanced bSSFP than for contrast-enhanced GRE
Keywords Balanced steady-state free precession (bSSFP) - Magnetic resonance imaging (MRI) · Hepatic veins - Portal venous anatomy · Liver surgery

\section{Introduction}

Breath-hold, dynamic two-dimensional (2D) and threedimensional (3D) gradient-echo (GRE) magnetic resonance imaging (MRI) using extracellular gadolinium-based contrast agents have emerged as accurate techniques for detection and characterization of focal liver lesions and assessment of the hepatic vasculature in patients with liver disease [1-3]. However, visualization of portal and hepatic veins by contrast-enhanced dynamic GRE imaging may be limited due to respiratory motion artifacts in patients with compromised respiratory function who are unable to hold their breath long enough several times in succession for dynamic GRE imaging [4-6]. In addition to dynamic 
contrast-enhanced GRE imaging, time-of-flight (TOF) sequences have been demonstrated to allow accurate assessment of portal and hepatic veins in patients with liver disease with and without the use of intravenous contrast medium $[7,8]$. However, TOF imaging is time consuming and limited in patients who have difficulties in holding their breath.

Balanced steady-state free precession (bSSFP) sequences are known to provide high signal from tissues with large ratios of $\mathrm{T} 2$ to $\mathrm{T} 1$ relaxation times, as may be found in blood, fluid, and fat, without the need of intravenous contrast medium. The introduction of fatsignal suppression in bSSFP imaging allows nonenhanced, flow-independent, visualization of the abdominal vasculature $[9,10]$. In a preliminary study with 11 patients, Wilson et al. [4] showed the feasibility of visualization of portal and hepatic veins by fat-suppressed, respiratory-triggered 2D bSSFP imaging. In ten patients who underwent right lobectomy of the liver, Carr et al. [5] depicted all accessory right hepatic veins on preoperative bSSFP imaging compared with the intraoperative findings.

The purpose of this study was a prospective intraindividual comparison of nonenhanced and contrast-enhanced fat-suppressed respiratory-triggered 2D bSSFP imaging with breath-hold contrast-enhanced dynamic 2D GRE and 2D TOF imaging for visualization of portal and hepatic veins and for assessment of surgically important portal and hepatic venous anatomical variants in patients with liver disease. When available, contrast-enhanced multidetectorrow computed tomography (MDCT) was used as the standard of reference.

\section{Materials and methods}

Patients

During a 6-month period, 60 consecutive patients with liver disease who underwent MRI of the liver for clinical indications were prospectively included into the study. Clinical indications for MRI were characterization of focal liver lesions in $42 / 60$ patients $(70 \%)$ and preoperative assessment of the liver before liver transplantation in $18 / 60$ patients $(30 \%)$. Liver cirrhosis was present in 32 of 60 patients $(53 \%)$. The underlying cause of liver cirrhosis was hepatitis $\mathrm{C}$ in 18 patients, alcohol abuse in six, hepatitis B in five, and hepatitis B combined with hepatitis C in three. Exclusion criteria for participation were history of an adverse reaction to paramagnetic contrast media besides general contraindications for MRI. During the study period, none of the 60 patients were excluded. Hence, the study group consisted of 60 adult patients (39 men with a mean age of 55 years, range 35-72 years; 21 women with a mean age of 46 years, range 23-70 years).

A contrast-enhanced MDCT examination of the liver during the portal venous phase was available in $40(67 \%)$ patients (24 men with a mean age of 52 years, range $41-$ 76 years; 16 women with a mean age of 52 years, range 23 70 years). Twenty-two of the 40 patients $(55 \%)$ underwent MDCT imaging before (mean, 120 days; range, 22229 days) and 18/40 (45\%) underwent MDCT after (mean, 80 days; range, 9-189 days) MRI. MDCT data sets of these 40 patients were used as standard of reference for detection of surgically important anatomical variants of portal and hepatic veins as well as presence of portal venous thrombosis.

The study was approved by the hospital's Institutional Review Board, and informed consent was obtained from all patients.

\section{MRI}

In all 60 patients, MRI was performed using a 1.5-T MR system (Signa EchoSpeed Plus, GE Healthcare, Milwaukee, WI, USA). Patients were positioned supine and feet first on the scan table with their arms placed above the head. An anteroposterior eight-element phased-array surface coil for signal reception was placed around the patient, covering the entire liver in all patients. A respiratory belt was placed around the patient's upper abdomen for respiratory triggering.

In addition to the sequences evaluated for the purpose of the study (see below), the imaging protocol of the liver in

Table 1 Imaging parameters of each different magnetic resonance sequence acquired in 60 patients with liver disease

\begin{tabular}{lllll}
\hline & $\begin{array}{l}\text { Nonenhanced bSSFP } \\
\text { Respiration-Triggering }\end{array}$ & $\begin{array}{l}\text { Contrast-enhanced GRE } \\
\text { Breath-holding }\end{array}$ & $\begin{array}{l}\text { Contrast-enhanced bSSFP } \\
\text { Respiration-Triggering }\end{array}$ & $\begin{array}{l}\text { Contrast-enhanced TOF } \\
\text { Breath-holding }\end{array}$ \\
\hline Time of repetition & $4.2 \mathrm{~ms}$ & $150 \mathrm{~ms}$ & $4.2 \mathrm{~ms}$ & $15.5 \mathrm{~ms}$ \\
Time of echo & $1.8 \mathrm{~ms}$ & $1.6 \mathrm{~ms}$ & $1.8 \mathrm{~ms}$ & $2.8 \mathrm{~ms}$ \\
Receiver bandwidth & $\pm 83 \mathrm{kHz}$ & $\pm 15 \mathrm{kHz}$ & $\pm 83 \mathrm{kHz}$ & $\pm 15 \mathrm{kHz}$ \\
Flip angle & $60^{\circ}$ & $80^{\circ}$ & $60^{\circ}$ & $60^{\circ}$ \\
Matrix & $256 \times 224$ & $256 \times 224$ & $256 \times 224$ & $256 \times 224$ \\
Slice thickness & $5 \mathrm{~mm}$ & $3 \mathrm{~mm}$ & $5 \mathrm{~mm}$ & $5 \mathrm{~mm}$ \\
Field of view & $40 \times 40$ & $40 \times 40$ & $40 \times 40$ & $40 \times 40$ \\
\hline
\end{tabular}

bSSFP balanced steady-state free precession, GRE gradient echo, TOF time of flight 
our institution included a transverse T2-weighted fast spin echo sequence with fat saturation, transverse T1-weighted fast multiplanar spoiled gradient recalled echo sequences in and out of phase, as well as a T2-weighted single shot fast spin echo sequence in the coronal plane without contrast medium application.

The imaging parameters of the different MR sequences used for the purpose of the study are summarized in Table 1. First, in all 60 patients, data from a nonenhanced fat-suppressed (using a spectrally fat-selective inversion pulse) 2D bSSFP sequence [fast imaging employing steady-state acquisition (FIESTA); GE Healthcare's bSSFP version] in the transverse plane was obtained. Subsequently, images from a transverse dynamic fatsaturated T1-weighted 2D fast GRE sequence [fast spoiled gradient-echo sequence (FSPGR)], a fat-suppressed 2D bSSFP sequence, as well as a 2D fast TOF sequence were acquired in all 60 patients after intravenous application of gadolinium diethylene triamine pentaacetic acid (GdDTPA) (Magnevist, Schering AG, Berlin, Germany). GdDTPA was administered through a 20- to 22-gauge needle placed in an antecubital vein at a dose of $0.1 \mathrm{mmol} / \mathrm{kg}$ body weight and at a flow rate of $2 \mathrm{ml} / \mathrm{s}$ using a power injector (Spectris, Medrad, Indianola, PA, USA). The contrast bolus was followed by a $20-\mathrm{ml}$ saline flush administered at the same flow rate.

Data from the GRE sequence were obtained in the hepatic arterial phase, the portal venous phase (60-80 s after contrast medium administration), and the equilibrium phase (180-240 s after contrast medium administration). Optimal delay times for the hepatic arterial phase were determined individually for each patient by using a test bolus of $2 \mathrm{ml} \mathrm{Gd-DTPA}$ and a multiphase sagittal singlesection gradient-recalled-echo sequence (repetition time, $5 \mathrm{~ms}$; echo time, $1 \mathrm{~ms}$; flip angle, $60^{\circ}$ ). Following the dynamic contrast-enhanced GRE MRI, first, the 2D bSSFP sequence was repeated and then the 2D TOF images were acquired without administration of a second contrast medium bolus in all patients. In all patients, GRE and TOF images were acquired during maximal inspiration. Balanced SSFP imaging was performed with respiratory triggering. The signal from the respiratory belt was used to trigger the start of the bSSFP sequence at the beginning of the end-expiration phase, which eliminated the need for respiration suspension. Since one image was acquired per breathing cycle, acquisition time of the respiratory synchronized sequences was given by the product of the total number of slices times the breathing-cycle period (range, $2 \mathrm{~min} 10 \mathrm{~s}$ to $2 \mathrm{~min} 40 \mathrm{~s}$; trigger point and trigger window at 20\%). In contrast, imaging time of the GRE sequences corresponded to a single breath-hold period (range, 25-30 s) whereas that of the TOF sequences corresponded to multiple 14-s breath-hold periods (range of imaging time, $2 \min 30 \mathrm{~s}$ to $2 \min 45 \mathrm{~s}$ ).

\section{MDCT imaging}

In all 40 patients who underwent MDCT for clinical reasons, MDCT scans were obtained with a 16-detectorrow CT scanner (Sensation 16; Siemens, Forchheim, Germany). Contrast-enhanced MDCT scanning was performed during portal venous phase (60-80 s after contrast medium application) after intravenous injection of $120 \mathrm{ml}$ nonionic iodinated contrast medium (iodixanol; Visipaque; Amersham Health, Buckinghamshire, England; $270 \mathrm{mg}$ iodine per milliliter) via a 20- to 22-gauge needle, which had been placed into a superficial vein in the antecubital fossa. The contrast medium was administered with an automated injector (Ulrich Medical AG, Ulm-Jungingen, Germany) at a flow rate of $4 \mathrm{ml} / \mathrm{s}$ followed by a $30-\mathrm{ml}$ flush of saline administered at the same flow rate. Data acquisition was performed with a nominal section thickness of $1.5 \mathrm{~mm}$, a table feed of $20 \mathrm{~mm}$ per rotation, and a 0.5 -s gantry rotation time (pitch, 0.8 ). X-ray tube voltage setting was $120 \mathrm{kV}$ at a mean tube current of $160 \mathrm{~mA}$. Transverse sections were reconstructed with a slice thickness of $2 \mathrm{~mm}$ at an interval of $1 \mathrm{~mm}$. The reconstruction field of view was $30 \mathrm{~cm}$ at a matrix size of $512 \times 512$.

\section{Image analysis}

Images were quantitatively and qualitatively analyzed on a dedicated interactive workstation (Advantage Windows Workstation 4.2, GE Healthcare, Buc, France).

\section{Quantitative analysis of MRI}

In all 60 patients, contrast-to-noise ratios (CNRs) were measured by one author of the study who was blinded to all patient data and who was not involved in the further course of the study. Quantitative image analysis was performed randomly with regard to patients order and MRI series. In addition, the different MRI series of each patient were analyzed in random order. Vessel to adjacent liver parenchyma CNRs were measured for the main (MPV), right (RPV), and left (LPV) portal vein, for the intrahepatic inferior vena cava (IVC), and for the largest of the three hepatic veins [right (RHV), middle (MHV), or left (LHV)]. To this end, reader-defined regions of interest (ROIs) were placed in the respective vessel, the adjacent liver parenchyma, and an image region in the air adjacent to the body within the coil. In the hepatic veins, ROIs were placed $1 \mathrm{~cm}$ proximal to the IVC. Once one of the three hepatic veins had been chosen for CNR measurement, the same vein was evaluated in all MRI series. Identical ROIs were used for all MRI series of a given patient unless slight positional changes due to respiration or patient movement required minimum adjustment of the ROIs. The ROIs were set to encompass as much as possible of each vessel (mean, 
$30 \mathrm{~mm}^{2}$; range, $\left.10-90 \mathrm{~mm}^{2}\right)$. CNR values were calculated as follows: $\mathrm{CNR}=$ (mean signal intensity in the vessel mean signal intensity in the adjacent liver parenchyma)/ standard deviation (SD) of the magnitude background signal in the air adjacent to the body within the coil.

\section{Qualitative analysis of MRI}

Subjective MRI quality of the MPV, RPV, LPV, intrahepatic IVC, and the three hepatic veins (RHV, MHV, and LHV together) was assessed for all sequence types in a consensus reading by two radiologists (readers 1 and 2) experienced in abdominal MRI. All MRIs of all 60 patients were available on the interactive workstation. The readers were blinded to all clinical data, and image analysis was performed randomly with regard to patients and MRI series. Both readers were allowed to individually adjust window centers and level settings for image analysis. A cine mode was available for rapid interactive interpretation. In addition, both readers were allowed to use transverse thick-slab maximum intensity projections (MIPs) of the MR data sets if considered useful. The image quality of each vessel was graded on a 5-point Likert scale: 1, not visible (no diagnostic information can be obtained from the images); 2, poor visibility (image quality of vessel is degraded due to low signal intensity and blurring artifacts); 3 , moderate visibility (image quality of vessel is degraded due to low signal intensity or blurring artifacts); 4, good visibility (high signal intensity and slight blurring artifacts); and 5, excellent visibility (high signal intensity and no blurring artifacts).

In 40 patients who also underwent MDCT imaging, readers 1 and 2 independently evaluated each MRI series with regard to the presence of surgically important anatomical variants of the intrahepatic portions of portal and hepatic veins. Patients as well as the MRI series were presented in random order. To minimize recall bias, only one MRI series was evaluated per reading session, which were separated by 3 weeks. The number and branching patterns of the RHV, MHV, and LHV were noted. The presence of three hepatic veins with a common trunk of the MHV and LHV on the IVC was considered the standard anatomy of the hepatic veins. Special attention was given to the presence of large $(>3 \mathrm{~mm})$ accessory RHV draining segments V to VIII directly into the IVC or into the MHV and to the presence of supernumerary LHVs [11]. In addition, presence of a separate drainage of the MHV and LHV into the IVC was noted. Simple bifurcation of the MPV into the RPV and the LPV with the RPV dividing into the right anterior and right posterior branches was considered as the standard anatomy of the portal vein [12]. Special attention was paid to the presence of a trifurcation of the MPV.

Finally, readers 1 and 2 independently notified the presence of a thrombus in the MPV, RPV, or LPV in each
MRI series of those 40 patients who also underwent MDCT imaging.

\section{Analysis of MDCT imaging}

All contrast-enhanced MDCT images were evaluated by a consensus panel consisting of one radiologist (reader 3) and two surgeons with experience in liver surgery (readers 4 and 5) on the basis of the transverse MDCT source data available on the interactive workstation (Advantage Windows Workstation 4.2). Readers were allowed to individually adjust window centers and level settings of MDCT images and use transverse thick-slab MIPs of the MDCT data sets, if considered useful. All MDCT data sets were assessed in random order, and the readers were blinded to all patient data, including results from MRI. For assessment of surgically important portal and hepatic venous anatomical variants, the same classification was used as for the evaluation of the MRI series. One reader (reader 3) measured the diameter of the accessory hepatic veins with an electronic caliper. In addition, readers 3-5 were asked to notify the presence of a thrombus in the MPV, RPV, or LPV based on a consensus reading of the MDCT scans.

\section{Statistical analysis}

The differences between nonenhanced bSSFP and contrastenhanced GRE, bSSFP, and TOF with regard to CNRs and subjective image quality were evaluated with the Wilcoxon signed rank test. A comparisonwise $p$ value of .05 or less was considered to indicate a statistically significant difference. CNRs and grading of subjective image quality are presented as means \pm SDs. Total sensitivities, specificities, positive and negative predictive values, and accuracy of each MRI series compared with contrastenhanced MDCT with regard to detection of surgically important portal and hepatic venous anatomical variants were calculated for each reader separately (readers 1 and 2) in 40 patients. To address dependencies of image analysis within the same patient, 95\% confidence intervals (CI) were calculated by using the proportion procedure for survey data of Stata (StataCorp, College Station, TX, USA) with the patient as primary sample unit. With regard to detection of surgically important portal and hepatic venous anatomical variants, differences between sensitivities and accuracy between each MRI series were assessed for each reader with the Wilcoxon signed rank test and using MDCT as the standard of reference. Total interobserver agreements between readers 1 and 2 for depiction of surgically important portal and hepatic venous anatomical variants were determined by calculating $\mathrm{K}$ values for each MRI series (poor, $\mathrm{k}=0$; slight, $\mathrm{\kappa} 0.01-0.2$; fair, $\mathrm{\kappa}$ 0.21-0.40; 
moderate, $\kappa$ 0.41-0.60; good, к 0.61-0.80; and excellent, K 0.81-1.00 agreement [13].

\section{Results}

Quantitative image analysis

Table 2 summarizes the means \pm SDs of CNRs as measured for portal and hepatic veins for each different MRI series. CNRs were statistically higher on contrast-enhanced bSSFP compared with nonenhanced bSSFP images in portal and hepatic veins (all $p<0.03$ ). CNRs for portal and hepatic veins on nonenhanced bSSFP were statistically higher than on contrast-enhanced GRE images (all $p<0.02$ ). CNRs were not statistically different for portal and hepatic veins on nonenhanced bSSFP compared with contrast-enhanced TOF images (all $p>0.1$ ).

Qualitative image analysis

Table 3 summarizes means \pm standard deviations of the subjective image quality of portal and hepatic veins as assessed on nonenhanced bSSFP and contrast-enhanced GRE, bSSFP, and TOF images. Subjective image quality of portal and hepatic veins was rated significantly higher by both readers on nonenhanced bSSFP compared with contrast-enhanced GRE (all $p<0.03$ ) and TOF (all $p<0.003$ ) (Fig. 1). On contrast-enhanced bSSFP, subjective image quality of portal and hepatic veins was rated significantly higher than that of nonenhanced bSSFP images by both readers (all $p<0.003$ ).

Findings regarding surgically important anatomical variants of intrahepatic portions of hepatic and portal veins as independently assessed by readers 1 and 2 on nonenhanced bSSFP, contrast-enhanced GRE, bSSFP, and TOF MRI, as well as by a consensus reading of readers $3-5$ on MDCT imaging in 40 patients are summarized in Table 4. On MDCT images, 46 accessory RHVs and LHVs with a mean diameter of $5.7 \mathrm{~mm}$ (range, $4.0-8.2 \mathrm{~mm}$ ) were identified in $22 / 40$ patients (55\%) (Figs. 1, 2 and 3). In ten of these 22 patients $(45 \%)$ there were two or more accessory veins. Separate drainage of the MHV and LHV into the IVC was present in $10 / 40$ patients $(25 \%)$. Portal vein trifurcation was identified in $4 / 40$ patients $(10 \%)$ (Fig. 4).

On both nonenhanced and contrast-enhanced bSSFP images, reader 1 missed one tributary vein from segment VIII and reader 2 missed one accessory vein from segment $\mathrm{V}$ and one tributary vein from segment VIII. Both readers depicted all accessory left veins and all separate drainage of the MHV and LHV into the IVC based on nonenhanced and contrast-enhanced bSSFP images. On contrast-enhanced GRE, reader 1 missed four tributary veins from segment VIII whereas reader 2 missed one accessory vein from segment V (Fig. 2), two accessory veins from segment VI (Fig. 3), one tributary vein from segment VII, and three tributary veins from segment VIII. In addition, reader 2 missed one separate drainage of the MHV and LHV into the IVC. On contrast-enhanced TOF images, reader 1 did not register three tributary veins from segment VIII; reader 2 missed one accessory vein from segment $\mathrm{V}$ and VI, respectively, and three tributary veins from segment VIII. Furthermore, reader 2 did not notify a separate drainage of the MHV and LHV into the IVC in one patient. Table 5 summarizes sensitivities, specificities, positive and negative predictive values, and accuracy of readers 1 and 2 compared with MDCT for each MRI series in the detection of surgically important portal and hepatic venous anatomical variants. For both readers, accuracy in comparison with MDCT reached $97 \%$ or more. There were no significant differences in sensitivity and accuracy between the MRI series for both readers with regard to detection of surgically important portal and hepatic venous anatomical variants $(p>0.1)$.

There was excellent agreement between readers 1 and 2 in detection of surgically important portal and hepatic venous anatomical variants on all MRI series, including nonenhanced bSSFP $(\kappa=0.97)$, contrast-enhanced GRE

Table 2 Measurements of contrast-to-noise ratios (CNRs) for portal and hepatic veins on magnetic resonance imaging (MRI) of 60 patients with liver disease acquired with different MRI sequences

\begin{tabular}{llllllll}
\hline \multirow{2}{*}{ Vessel } & \multicolumn{2}{l}{ Contrast-to-noise ratios } & & & \\
\cline { 2 - 8 } & $\begin{array}{l}\text { Nonenhanced } \\
\text { bSSFP }\end{array}$ & $\begin{array}{l}\text { Contrast-enhanced } \\
\text { GRE }\end{array}$ & $\begin{array}{l}p \\
\text { value }\end{array}$ & $\begin{array}{l}\text { Contrast-enhanced } \\
\text { bSSFP }\end{array}$ & $\begin{array}{l}p \\
\text { value }\end{array}$ & $\begin{array}{l}\text { Contrast-enhanced } \\
\text { TOF }\end{array}$ & $\begin{array}{l}p \\
\text { value }\end{array}$ \\
\hline Main portal vein & $29.3 \pm 14.4$ & $25.7 \pm 14.8$ & .01 & $34.1 \pm 14.8$ & .02 & $27.2 \pm 2.6$ \\
Right portal vein & $31.8 \pm 19.7$ & $25.0 \pm 17.3$ & .01 & $39.7 \pm 31.3$ & .03 & $29.0 \pm 7.6$ \\
Left portal vein & $29.8 \pm 12.7$ & $20.7 \pm 15.0$ & .008 & $33.7 \pm 17.2$ & .006 & $21.9 \pm 19.0$ \\
Inferior Vena & $34.0 \pm 11.5$ & $24.5 \pm 20.6$ & .01 & $36.0 \pm 13.1$ & .01 & $19.2 \pm 11.1$ & 0.4 \\
Cava & & $18.4 \pm 14.0$ & .02 & $16.0 \pm 11.0$ & 0.9 & .008 & $12.9 \pm 10.3$ \\
Hepatic Vein & $14.0 \pm 7.9$ & & & & 0.1 \\
\hline
\end{tabular}

Numbers are means \pm standard deviations; $p$ values are calculated compared with nonenhanced bSSFP

bSSFP balanced steady-state free precession, GRE, gradient echo, TOF time of flight 
Table 3 Assessment of image quality of portal and hepatic veins on magnetic resonance imaging (MRI) of 60 patients with liver disease, acquired with different MRI sequences

\begin{tabular}{llllllll}
\hline \multirow{2}{*}{\begin{tabular}{l} 
Vessel \\
\cline { 2 - 7 }
\end{tabular}} & $\begin{array}{l}\text { Image quality } \\
\text { bSSFP }\end{array}$ & $\begin{array}{l}\text { Contrast-enhanced } \\
\text { GRE }\end{array}$ & $\begin{array}{l}p \\
\text { value }\end{array}$ & $\begin{array}{l}\text { Contrast-enhanced } \\
\text { bSSFP }\end{array}$ & $\begin{array}{l}p \\
\text { value }\end{array}$ & $\begin{array}{l}\text { Contrast-enhanced } \\
\text { TOF }\end{array}$ & $\begin{array}{l}p \\
\text { value }\end{array}$ \\
\hline Main portal vein & $3.5 \pm 0.7$ & $3.1 \pm 0.6$ & .02 & $3.7 \pm 0.7$ & .001 & $2.6 \pm 0.9$ \\
Right portal vein & $3.5 \pm 0.7$ & $3.1 \pm 0.6$ & .02 & $3.7 \pm 0.7$ & .001 & $2.6 \pm 0.9$ & .002 \\
Left portal vein & $3.5 \pm 0.7$ & $3.1 \pm 0.6$ & .01 & $3.6 \pm 0.7$ & .002 & $2.4 \pm 0.9$ \\
Inferior vena & $3.4 \pm 0.9$ & $3.0 \pm 0.6$ & .03 & $3.5 \pm 0.6$ & .003 & $2.4 \pm 0.9$ & .002 \\
$\begin{array}{l}\text { cava } \\
\text { Hepatic vein }\end{array}$ & $3.3 \pm 1.2$ & $3.1 \pm 0.6$ & .01 & $3.4 \pm 0.9$ & .001 & $2.4 \pm 0.9$ \\
\hline
\end{tabular}

Numbers are means \pm standard deviations; $p$ values are calculated compared with nonenhanced bSSFP $b S S F P$ balanced steady-state free precession, GRE gradient echo, TOF time of flight

( $K=0.89)$, contrast-enhanced bSSFP $(\kappa=0.97)$, and contrast-enhanced TOF $(\kappa=0.92)$. No thrombus was notified by readers $3-5$ in MPV, RPV, or LPV based on the MDCT images obtained in 40 patients. There was no thrombus noted in the MPV, RPV, or LPV on any MRI series as assessed by readers 1 and 2 in the same 40 patients.
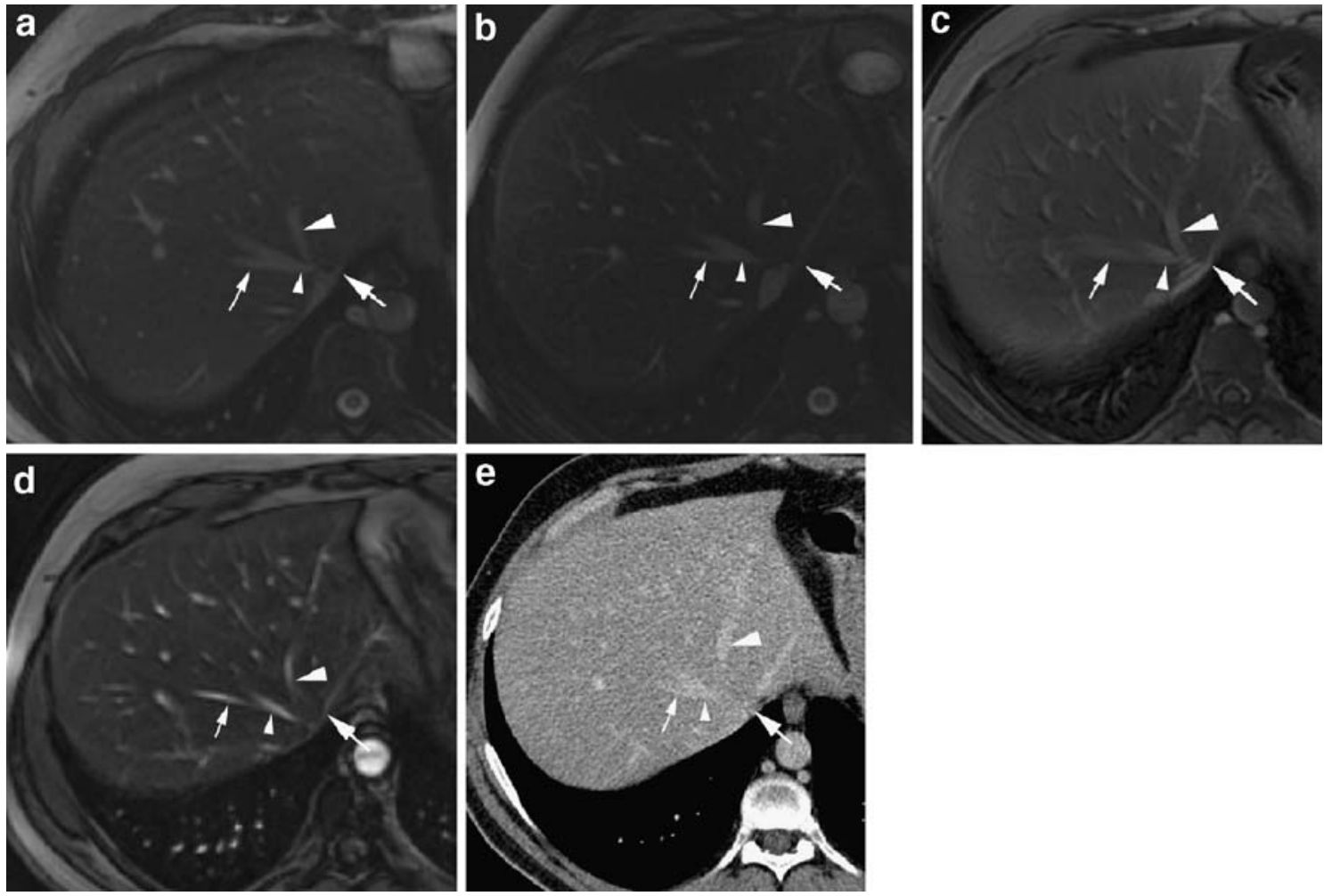

Fig. 1 a Transverse nonenhanced balanced steady-state free precession (bSSFP) image of the liver in a 35-year-old man at the level of the middle (MHV; small arrowhead) and left (LHV; large arrowhead) hepatic veins demonstrates accessory hepatic vein from segment VIII (small arrow) draining into the MHV. In addition, accessory LHV (large arrow), which drains directly into the inferior vena cava (IVC) can be seen. Image quality of MHV, LHV, and IVC was rated as excellent by both readers. Note common trunk of MHV and LHV. b On contrast-enhanced bSSFP image obtained in the same patient, image quality of MHV, LHV, and IVC was rated as excellent by both readers. Both readers also noted accessory hepatic vein from segment VIII and accessory LHV. c Both readers graded image quality of MHV and IVC as moderate and image quality of LHV as good on contrast-enhanced GRE image in the same patient. Both readers also noted accessory hepatic vein from segment VIII and accessory LHV. d Due to slight blurring artifacts, image quality of MHV, LHV, and IVC was rated as good by both readers on contrast-enhanced time-of-flight (TOF) image of the liver in the same patient. Accessory hepatic vein from segment VIII and accessory LHV were also noted by both readers. e Multidetectorrow computed tomography (MDCT) of the same patient confirmed presence of accessory hepatic vein from segment VIII and accessory LHV. Note that the common trunk of MHV and LHV is not visible on images b-e since this part extends out of the image plane 
Table 4 Numbers of surgically important anatomical variants of portal and hepatic veins identified on images obtained by multidetectorrow computed tomography (MDCT) and different magnetic resonance imaging (MRI) sequences in 40 patients with liver disease

\begin{tabular}{|c|c|c|c|c|c|c|c|c|c|}
\hline \multirow[t]{2}{*}{ Anatomical variation } & \multirow{2}{*}{$\begin{array}{l}\text { MDCT } \\
\text { Readers 4-6 }\end{array}$} & \multicolumn{2}{|c|}{ Nonenhanced bSSFP } & \multicolumn{2}{|c|}{ Contrast-enhanced GRE } & \multicolumn{2}{|c|}{ Contrast-enhanced bSSFP } & \multicolumn{2}{|c|}{ Contrast-enhanced TOF } \\
\hline & & Reader 2 & Reader 3 & Reader 2 & Reader 3 & Reader 2 & Reader 3 & Reader 2 & Reader 3 \\
\hline Segment V & 6 & 6 & 5 & 6 & 5 & 6 & 5 & 6 & 5 \\
\hline Segment VI & 12 & 12 & 12 & 12 & 10 & 12 & 12 & 12 & 11 \\
\hline Segment VII & 4 & 4 & 4 & 4 & 3 & 4 & 4 & 4 & 4 \\
\hline Segment VIII & 18 & 17 & 17 & 14 & 15 & 17 & 17 & 15 & 15 \\
\hline 2 LHV & 6 & 6 & 6 & 6 & 6 & 6 & 6 & 6 & 6 \\
\hline Separate MHV/LHV & 10 & 10 & 10 & 10 & 9 & 10 & 10 & 10 & 9 \\
\hline Portal trifurcation & 4 & 4 & 4 & 4 & 4 & 4 & 4 & 4 & 4 \\
\hline
\end{tabular}

bSSFP balanced steady-state free precession, GRE gradient echo, TOF time of flight, $L H V$ left hepatic vein, $M H V$ middle hepatic vein

\section{Discussion}

Accurate preoperative assessment of the anatomy and patency of portal and hepatic veins in patients with liver disease is of paramount importance for successful liver surgery. Several imaging modalities allow preoperative evaluation of portal and hepatic veins in patients with liver disease. Catheter digital subtraction angiography (DSA), including indirect portography, has been considered the standard of reference for assessment of arterial and portal venous anatomy. However, morbidity and mortality associated with this imaging modality combined with its limitations in the depiction of the hepatic venous anatomy have prompted the development of alternative noninvasive imaging procedures. Duplex ultrasonography (US), MDCT, and MRI have been shown to be valuable noninvasive alternatives for evaluation of portal and hepatic veins. Duplex US allows a noninvasive evaluation of portal and hepatic veins, including assessment of blood velocity and flow directions. However, duplex US is reader dependent and time consuming, and obese patients or patients unable to hold their breaths are difficult to examine
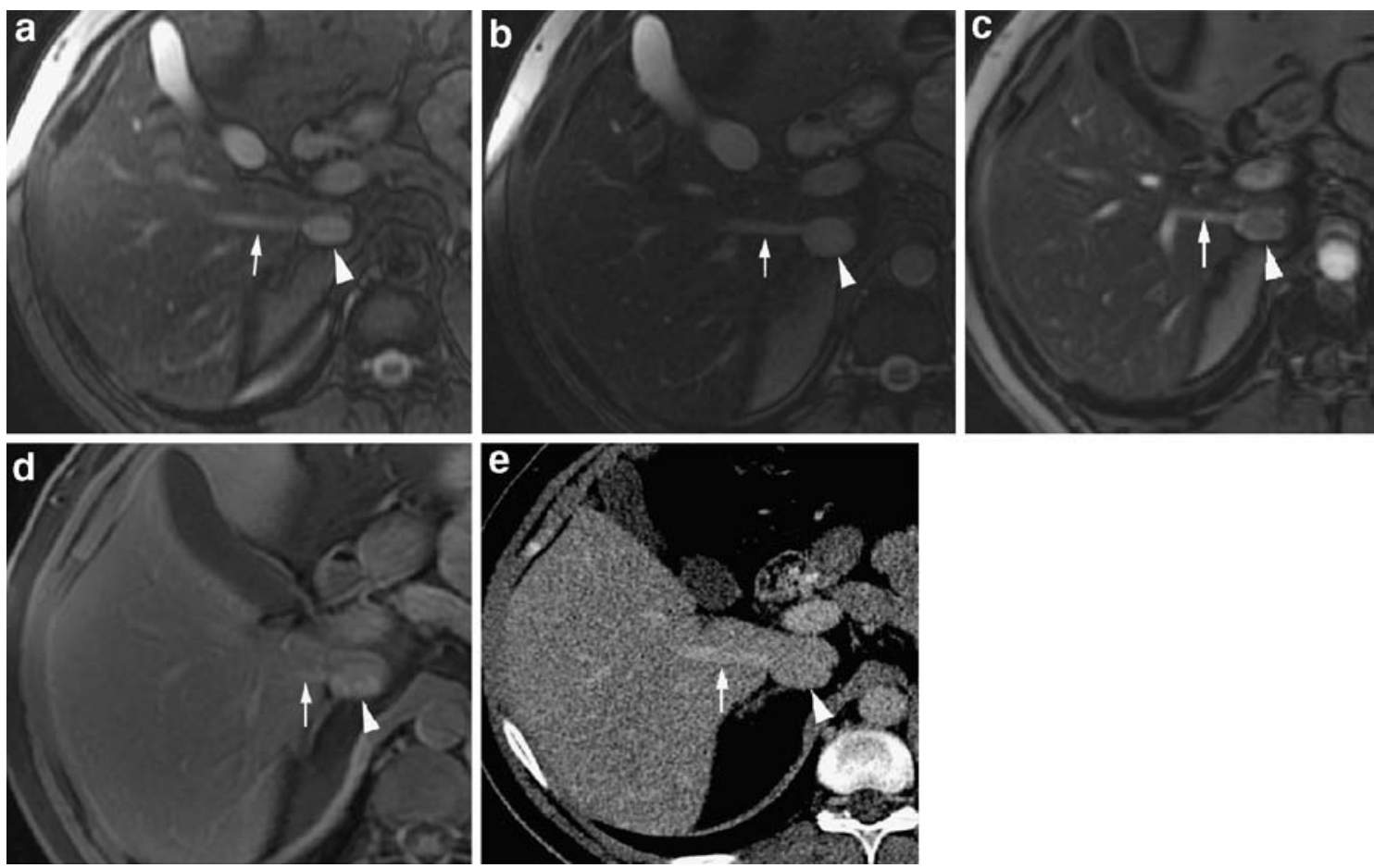

Fig. 2 Accessory hepatic vein (arrow) draining segment V directly into the inferior vena cava (IVC) (arrowhead) in a 45-year-old man. Both readers depicted accessory hepatic vein draining segment $\mathrm{V}$ (arrow) on nonenhanced balanced steady-state free precession (bSSFP) (a), contrast-enhanced bSSFP (b), and contrast-enhanced time-of-flight (TOF) (c) images. On contrast-enhanced gradient-

echo (GRE) image (d), presence of accessory hepatic vein draining segment $\mathrm{V}$ was missed by reader 2 . Multidetector-row computed tomography (MDCT) (e) obtained in the same patient confirmed presence of accessory hepatic vein (arrow) draining segment $\mathrm{V}$ into the IVC (arrowhead) 

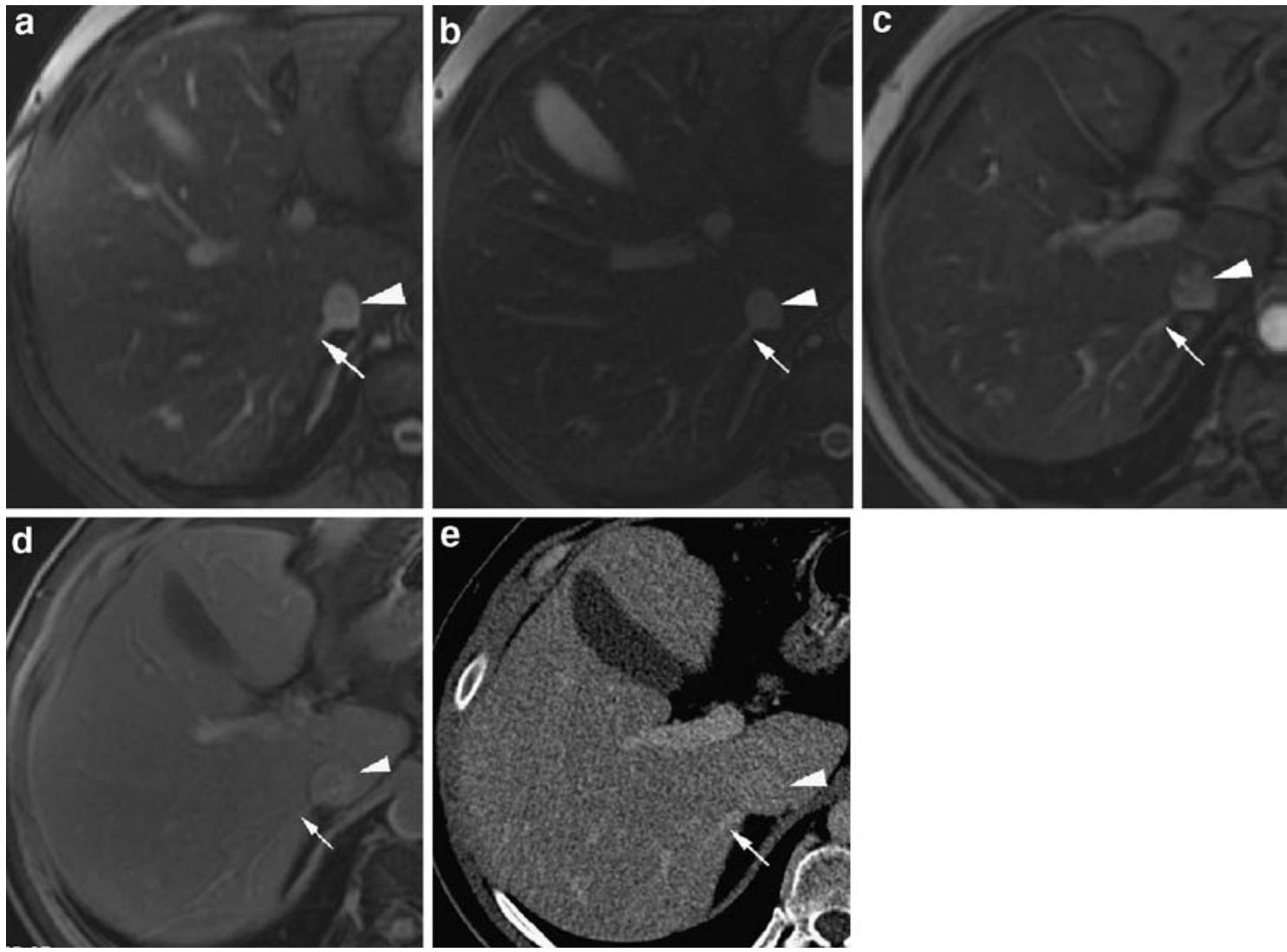

Fig. 3 A 64-year-old woman with accessory hepatic vein draining segment VI (arrow) directly into the inferior vena cava (IVC) (arrowhead). On nonenhanced balanced steady-state free precession (bSSFP) (a), contrast-enhanced bSSFP (b), and contrast-enhanced time-of-flight (TOF) (c) images, both readers noted the presence of accessory hepatic vein (arrow) draining segment VI into the IVC

preoperatively. In addition, duplex US does not provide a preoperative road map of portal and hepatic vein anatomy, which is considered of help for surgical planning.

Due to the fast imaging capabilities of MDCT scanners, a resolution of the intravenous contrast bolus passage into (arrowhead). Reader 2 missed accessory hepatic vein draining segment VI on contrast-enhanced gradient-echo (GRE) image (d). Presence of accessory hepatic vein (arrow) draining segment VI into the IVC (arrowhead) was confirmed on multidetector-row computed tomography (MDCT) (e) of the same patient

separate phases, including the hepatic arterial and portal venous phases, became possible. Several studies have demonstrated excellent correlation between MDCT imaging and catheter DSA or intraoperative sonography in assessment of portal and hepatic veins, with accuracy of up
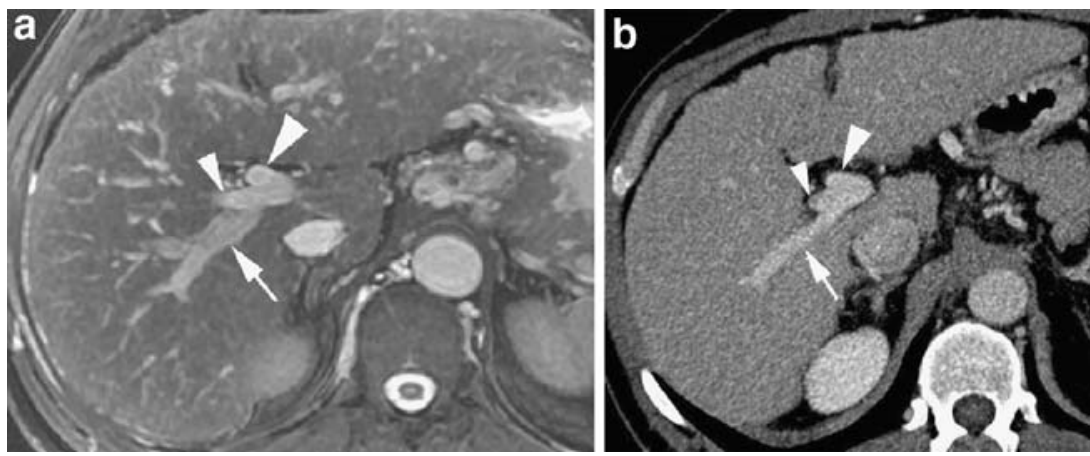

Fig. 4 A 42-year-old man with liver cirrhosis and portal-vein trifurcation. a Transverse thick-slab maximum intensity projection (MIP) of nonenhanced balanced steady-state free precession (bSSFP) image demonstrates portal vein trifurcation with common trunk of right anterior (small arrowhead) and posterior (arrow) branches as well as left portal vein (large arrowhead). b Presence of portal vein trifurcation was confirmed on transverse thick-slab MIP of multidetector-row computed tomography (MDCT) data set obtained in the same patient 
Table 5 Detection of surgically important anatomical variants of portal and hepatic veins in 40 patients with liver disease on images acquired with different magnetic resonance imaging (MRI) sequences using multidetector-row computed tomography (MDCT) as the standard of reference

\begin{tabular}{|c|c|c|c|c|c|c|c|c|}
\hline & \multicolumn{2}{|c|}{ Nonenhanced bSSFP } & \multicolumn{2}{|c|}{ Contrast-enhanced GRE } & \multicolumn{2}{|c|}{ Contrast-enhanced bSSFP } & \multicolumn{2}{|c|}{ Contrast-enhanced TOF } \\
\hline & Reader 2 & Reader 3 & Reader 2 & Reader 3 & Reader 2 & Reader 3 & Reader 2 & Reader 3 \\
\hline Sensitivity & $\begin{array}{l}98.3 \\
(94.9,100)\end{array}$ & $\begin{array}{l}96.7 \\
(92.1,100)\end{array}$ & $\begin{array}{l}93.3 \\
(87.5,99.1)\end{array}$ & $\begin{array}{l}86.7 \\
(78.7,94.7)\end{array}$ & $\begin{array}{l}98.3 \\
(94.9,100)\end{array}$ & $\begin{array}{l}96.7 \\
(92.1,100)\end{array}$ & $\begin{array}{l}95.0 \\
(89.8,100)\end{array}$ & $\begin{array}{l}90 \\
(82.2,97.8)\end{array}$ \\
\hline Specificity & $100(100,100)$ & $100(100,100)$ & $100(100,100)$ & $100(100,100)$ & $100(100,100)$ & $100(100,100)$ & $100(100,100)$ & $100(100,100)$ \\
\hline PPV & $100(100,100)$ & $100(100,100)$ & $100(100,100)$ & $100(100,100)$ & $100(100,100)$ & $100(100,100)$ & $100(100,100)$ & $100(100,100)$ \\
\hline Accuracy & $\begin{array}{l}99.6 \\
(98.8,100)\end{array}$ & $\begin{array}{l}99.3 \\
(98.3,100)\end{array}$ & $\begin{array}{l}98.6 \\
(97.4,99.8)\end{array}$ & $\begin{array}{l}97.1 \\
(95.1,99.1)\end{array}$ & $\begin{array}{l}99.6 \\
(98.8,100)\end{array}$ & $\begin{array}{l}99.3 \\
(98.3,100)\end{array}$ & $\begin{array}{l}98.9 \\
(97.7,100)\end{array}$ & $\begin{array}{l}97.9 \\
(96.1,99.7)\end{array}$ \\
\hline
\end{tabular}

Data in parentheses are $95 \%$ confidence intervals

$b S S F P$ balanced steady-state free precession, $G R E$ gradient echo, $T O F$ time of flight, $P P V$ positive predictive value, $N P V$ negative predictive value

to $100 \%$ for depiction of surgically important portal and hepatic venous anatomical variants [14-17]. However, MDCT with the use of iodinated contrast medium, is limited in patients with renal insufficiency. In addition, a major drawback of MDCT imaging is the potentially hazardous radiation exposure associated with this technique. A radiation dose between 15 and $20 \mathrm{mSv}$ has been estimated for a dedicated MDCT examination of the liver, which restricts the use of MDCT imaging, particular in healthy young potential living liver donors [18].

Breath-hold dynamic contrast-enhanced GRE imaging has been established as a safe, accurate, and noninvasive imaging technique for assessment of the hepatic vasculature without the use of ionizing radiation [1-3]. However, since preoperative assessment of the hepatic vasculature includes evaluation of the hepatic arterial supply apart from assessment of portal and hepatic veins, the first postcontrast MRI data acquisition is usually timed to coincide with the hepatic arterial phase of the contrast bolus. The following postcontrast MRI data acquisitions, therefore, may not be optimally synchronized with the portal venous contrast phases in some instances [4, 5]. Furthermore, patients with hepatic encephalopathy, ascites or cardiopulmonary diseases may not be able to repeatedly hold their breath, thereby limiting the technique's utility in patients with liver disease and compromised respiratory function. In general, the scan time in patients who cannot hold their breath long enough can be reduced by increasing the slice thickness, decreasing the number of phaseencoding steps, increasing the receiver bandwidth, and/or by parallel imaging. Most often, these options compromise image quality. Therefore, an additional approach is desired for dedicated assessment of portal and hepatic veins.

Fat-suppressed balanced bSSFP is a fast imaging technique with the capability of respiratory-triggering, which can also be performed in patients who cannot hold their breath several times in succession. The bSSFP sequence yields an image contrast that depends on ratios of $\mathrm{T} 2$ over $\mathrm{T} 1$ relaxation times and, thus, offers bright signal from blood and fat $[9,10]$. Therefore, this sequence can also be used for morphological vascular imaging without the need of intravenous contrast medium [10]. Wilson et al. [4] have shown that nonenhanced respiratorytriggered bSSFP imaging is accurate for assessment of the portal vein. Compared with contrast-enhanced CT, US, or catheter DSA as standards of reference, bSSFP imaging correctly depicted portal venous pathologies, including portal vein occlusion, stenosis, and tumor compression in ten out of 11 patients [4]. In a study with 24 patients, Carr et al. [5] demonstrated improved visualization of the portal vein and its branches on bSSFP images compared with contrast-enhanced dynamic GRE imaging. In all ten patients who underwent right lobectomy in this study, bSSFP imaging visualized accessory hepatic vein branches with sensitivity and specificity of $100 \%$, respectively, compared with intraoperative findings [5]. Amano et al. [19] also confirmed the potential of bSSFP imaging for assessment of portal and visceral veins in 20 patients with chronic liver disease or other types of abdominal organ disorders.

Our study demonstrated good feasibility of fat-suppressed bSSFP imaging for assessment of portal and hepatic veins in a large number of patients with and without liver cirrhosis. On average, subjective image quality of the different vessels of portal and hepatic veins was rated higher by both readers on bSSFP images than on contrast-enhanced GRE and TOF images.

The feasibility of bSSFP imaging for assessment of portal and hepatic veins is also reflected by the high CNR values measured for portal and hepatic veins. CNRs were significantly higher on bSSFP imaging compared with CNRs on GRE imaging. In addition, CNRs were significantly higher on contrast-enhanced than on nonenhanced bSSFP images. Furthermore, image quality of 
portal and hepatic veins was rated significantly higher on contrast-enhanced bSSFP than on nonenhanced bSSFP images by both readers. Since the center of k-space was measured in the 112th TR period, the contrast of our images is expected to closely resemble the steady-state signal contrast even though acquisition was synchronized with the breathing rhythm. Since visualization of blood in bSSFP imaging thus depends on the ratio of blood T2 to T1 relaxation times, CNRs in portal and hepatic veins are increased by a decrease of the $\mathrm{T} 1$ relaxation time of blood after intravenous administration of contrast medium, which is relatively larger than the decrease of the T2 time. Foo et al. [20] estimated that the contrast-enhanced bSSFP blood signal is higher than the nonenhanced blood bSSFP signal for up to 10 to $20 \mathrm{~min}$ after intravenous contrast medium administration. Therefore, to fully exploit the potential of bSSFP imaging, acquisition of the bSSFP sequence should be performed after intravenous administration of contrast medium in patients who undergo contrast-enhanced imaging of the liver.

There was no statistically significant difference between nonenhanced bSSFP imaging and contrast-enhanced TOF imaging with regard to the measured CNRs in portal and hepatic veins. However, subjective image quality of portal and hepatic veins as rated by both readers was significantly higher for bSSFP images than for contrast-enhanced TOF images due to smaller respiration artifacts. Compared with TOF imaging, bSSFP imaging can be performed with respiratory triggering and, therefore, may also be acquired in patients who are not able to repeatedly hold their breath. Thus, bSSFP imaging may be preferred to TOF imaging for assessment of portal and hepatic veins in patients with liver disease.

In particular, in living-donor liver transplantation, preoperative knowledge of vascular structures traversing the surgical plane is important to minimize vascular complications and provide good liver function. It has been increasingly recognized that right accessory hepatic veins larger than $3 \mathrm{~mm}$ and draining segments V to VIII should be anastomosed into the IVC of the recipient to prevent hepatic venous congestion of the liver graft [21]. Accordingly, in partial transplantation of left liver grafts, in particular in pediatric or small adult recipients, accurate preoperative depiction of anatomical variants of the outflow of segments II-IV (including absence of a common trunk of MHV and LHV or presence of an accessory LHV) is important for adequate outflow reconstruction of all hepatic veins to avoid early graft dysfunction [22]. Bench surgery with hepatic-vein venoplasty or the interposition of a venous graft from the living donor (e.g., saphenous vein or inferior mesenteric vein), cadaveric grafts or nonbiologic conduits have been used to drain paramedian segments (i.e., segment IV in left liver donation or segments V-VIII in right liver donation) [23, 24]. Based on MDCT imaging, accessory right and left hepatic veins larger than $3 \mathrm{~mm}$ were present in $55 \%$ of patients in our study. On nonenhanced and contrastenhanced bSSFP imaging, both readers detected accessory hepatic veins with an accuracy of $99 \%$ compared with MDCT imaging. The high level of reliability of bSSFP imaging in the detection of hepatic venous anatomical variants is reflected by excellent interobserver agreement in this study. Due to respiration artifacts, both readers missed four and eight accessory right and left hepatic veins, respectively, on contrast-enhanced GRE and three and six accessory right and left veins, respectively, on contrastenhanced TOF imaging. In addition, interobserver agreement with regard to depiction of variant portal and hepatic venous anatomy was lower on both contrast-enhanced GRE and TOF imaging compared with bSSFP imaging but was, however, still excellent. Separate drainage of the MHV and LHV into the IVC was present in ten of 40 patients $(25 \%)$ in our study and was correctly depicted on nonenhanced and contrast-enhanced bSSFP imaging by both readers. In one of ten patients, reader 2 missed the separate drainage of the MHV and LHV into the IVC on both contrast-enhanced GRE and TOF imaging.

Another important issue in living-donor liver transplantation is the portal venous anatomy of the partial graft. Since selection criteria for potential liver donors are very strict, some transplantation centers refuse donors with documented anatomical variants of the portal vein. Portal vein trifurcation has been reported in $4-16 \%$ of patients on CT, US, or intraoperative studies [10, 17-20]. In our study, portal vein trifurcation was present in four of 40 patients $(10 \%)$ based on MDCT imaging and was detected by both readers in all four patients on all MRI series.

The results of this study may have direct influence on the MRI protocol of patients with liver disease. In our daily clinical practice, bSSFP imaging is now used as an accurate complement for assessment of portal and hepatic veins in patients who cannot hold their breath long enough several times in succession. We obtain the bSSFP sequence at the end of our liver imaging protocol after administration of intravenous contrast medium since our study has demonstrated that both CNRs and image quality are higher on contrast-enhanced compared with nonenhanced bSSFP imaging.

The following limitations of our study need to be addressed. First, assessment of accuracy of bSSFP imaging with regard to detection of surgically important anatomical variants of portal and hepatic veins was only possible in the subgroup of 40 of 60 patients $(67 \%)$ with available contrast-enhanced MDCT of the liver. Second, we did not compare the $2 \mathrm{D}$ bSSFP sequence with a $3 \mathrm{D}$ GRE sequence, which has been shown to be valuable for assessment of portal and hepatic veins [1, 2]. However, for better comparison with the 2D bSSFP sequence used in our study, we chose a 2D sequence for GRE imaging with the same slice thickness, matrix, and field of view. In addition, a second MRI session with additional intravenous administration of contrast medium for comparison with a 
contrast-enhanced dynamic 3D GRE sequence was considered not justified. Future studies are needed to compare the performance of the bSSFP sequence with a contrastenhanced 3D GRE sequence for assessment of portal and hepatic veins. Finally, with the introduction of parallel imaging techniques reduction of the imaging time of GRE imaging can be achieved [6]. Further studies are warranted to demonstrate improved image quality of portal and hepatic veins on GRE imaging by using parallel imaging techniques in patients with problems holding their breath.
In conclusion, our study demonstrated in a prospective intraindividual comparison of patients with liver disease that fat-suppressed, respiratory-triggered 2D bSSFP imaging is useful for assessment of portal and hepatic veins. Compared with contrast-enhanced MDCT as a standard of reference, the presence of surgically important portal and hepatic venous anatomical variants can be predicted with high accuracy.

\section{References}

1. Ito K, Blasbalg R, Hussain SM, Mitchell DG (2000) Portal vein and its tributaries: evaluation with thin-section three-dimensional contrast-enhanced dynamic fat-suppressed MR imaging. Radiology 215:381-386

2. Lee VS, Lavelle MT, Rofsky NM, Laub G, Thomasson DM, Krinsky GA, Weinreb JC (2000) Hepatic MR imaging with a dynamic contrast-enhanced isotropic volumetric interpolated breath-hold examination: feasibility, reproducibility, and technical quality. Radiology 215:365-372

3. Lavelle MT, Lee VS, Rofsky NM, Krinsky GA, Weinreb JC (2001) Dynamic contrast-enhanced threedimensional MR imaging of liver parenchyma: source images and angiographic reconstructions to define hepatic arterial anatomy. Radiology 218:389-394

4. Wilson MW, LaBerge JM, Kerlan RK, Martin AJ, Weber OM, Roberts T, Vitalich C, Higgins CB, Gordon RL (2002) MR portal venography: preliminary results of fast acquisition without contrast material or breath holding. Acad Radiol 9:1179-1184

5. Carr JC, Nemcek AA Jr, Abecassis M, Blei A, Clarke L, Pereles FS, McCarthy R, Finn JP (2003) Preoperative evaluation of the entire hepatic vasculature in living liver donors with use of contrast-enhanced MR angiography and true fast imaging with steady-state precession. J Vasc Interv Radiol 14:441-449
6. McKenzie CA, Lim D, Ransil BJ, Morrin M, Pedrosa I, Yeh EN, Sodickson DK, Rofsky NM (2004) Shortening MR image acquisition time for volumetric interpolated breath-hold examination with a recently developed parallel imaging reconstruction technique: clinical feasibility. Radiology 230:589-594

7. Leyendecker JR, Rivera E Jr, Washburn WK, Johnson SP, Diffin DC, Eason JD (1997) MR angiography of the portal venous system: techniques, interpretation, and clinical applications. Radiographics 17:1425-1443

8. Hughes LA, Hartnell GG, Finn JP, Longmaid HE, Volpe J, Wheeler HG, Clouse ME (1996) Time-of-flight MR angiography of the portal venous system: value compared with other imaging procedures. AJR Am J Roentgenol 166:375-378

9. Hargreaves BA, Vasanawala SS, Nayak KS, Hu BS, Nishimura DG (2003) Fatsuppressed steady-state free precession imaging using phase detection. Magn Reson Med 50:210-213

10. Scheffler K, Lehnhardt S (2003) Principles and applications of balanced SSFP techniques. Eur Radiol 13:2409-2418

11. Varotti G, Gondolesi GE, Goldman J, Wayne M, Florman SS, Schwartz ME, Miller CM, Sukru E (2004) Anatomic variations in right liver living donors. J Am Coll Surg 198:577-582

12. Akgul E, Inal M, Soyupak S, Binokay F, Aksungur E, Oguz M (2002) Portal venous variations. Prevalence with contrast-enhanced helical CT. Acta Radiol 43:315-319
13. Landis JR, Koch GG (1977) An application of hierarchical kappa-type statistics in the assessment of majority agreement among multiple observers. Biometrics 33:363-374

14. Sahani D, Saini S, Pena C, Nichols S, Prasad SR, Hahn PF, Halpern EF, Tanabe KK, Mueller PR (2002) Using multidetector CT for preoperative vascular evaluation of liver neoplasms: technique and results. AJR Am J Roentgenol 179:53-59

15. Guiney MJ, Kruskal JB, Sosna J, Hanto DW, Goldberg SN, Raptopoulos V (2003) Multi-detector row CT of relevant vascular anatomy of the surgical plane in split-liver transplantation. Radiology 229:401-407

16. Stemmler BJ, Paulson EK, Thornton FJ, Winters SR, Nelson RC, Clary BM (2004) Dual-phase 3D MDCT angiography for evaluation of the liver before hepatic resection. AJR Am J Roentgenol 183:1551-1557

17. Takahashi S, Murakami T, Takamura M, Kim T, Hori M, Narumi Y, Nakamura H, Kudo M (2002) Multidetector row helical CT angiography of hepatic vessels: depiction with dualarterial phase acquisition during single breath hold. Radiology 222:81-88

18. Schroeder T, Malago M, Debatin JF, Goyen M, Nadalin S, Ruehm SG (2005) "All-in-one" imaging protocols for the evaluation of potential living liver donors: comparison of magnetic resonance imaging and multidetector computed tomography. Liver Transpl 11:776-787 
19. Amano Y, Takahama K, Nozaki A, Amano M, Kumazaki T (2004) Magnetic resonance portography using contrast-enhanced fat-saturated threedimensional steady-state free precession imaging. J Magn Reson Imaging 19:238-244
20. Foo TK, Ho VB, Marcos HB, Hood MN, Choyke PL (2002) MR angiography using steady-state free precession. Magn Reson Med 48:699-706

21. Russo MW, Brown RS Jr (2004) Adult living donor liver transplantation. Am J Transplant 4:458-465

22. Suh K (2004) Technical variations in living donor liver transplantation. Curr Opin Organ Transplant 9:90-98

23. Lee KW, Lee DS, Lee HH, Joh JW, Choi SH, Heo JS, Lee SK, Kim SJ (2004) Interpostion vein graft in living donor liver transplantation. Transplant Proc 36:2261-2262
24. Miller CM, Gondolesi GE, Florman S, Matsumoto C, Munoz L, Yoshizumi T, Artis T, Fishbein TM, Sheiner PA, Kim-Schluger L, Schiano T, Shneider BL, Emre S, Schwartz ME (2001) One hundred nine living donor liver transplants in adults and children: a singlecenter experience. Ann Surg 234:301-311 